\title{
A Second-Order Improved Front Tracking Method for the Numerical Treatment of the Hyperbolic Euler Equations
}

\author{
J.A.S. Witteveen
}

Summary. Front tracking methods can be used to accurately resolve discontinuities in numerical simulations of Euler flows. They usually result in first-order error convergence due to their piecewise constant approximation of the flow conditions. In this chapter, a piecewise linear reconstruction of the solution is proposed based on wave types which track the physical phenomena that the fronts represent. It is demonstrated numerically that this approach results in second-order error convergence. A verification and validation study is performed by comparing the results with those of the Godunov method and experimental data.

\section{Introduction}

Front tracking can be used as a numerical method for resolving discontinuities in the simulation of hyperbolic conservation laws. In this chapter, front tracking is considered as a tool for the numerical simulation of the Euler equations of inviscid gas dynamics. The Euler equations allow for discontinuous solutions such as shock waves, slip lines, and two-fluid interfaces. Front tracking is often used for resolving these discontinuities in addition to a background grid for resolving the continuous flow phenomena. Richtmyer and Morton [RM67] have initiated this branch of front tracking methods. Important contributions and applications have been presented by Moretti [Mor87], Swartz and Wendroff [SW86], and Glimm, Grove, Chern, Holmes, and coworkers [CGM86, HGS95].

Front tracking can also be used for resolving both the discontinuous and continuous flow phenomena. In that case the continuous phenomena are approximated by a piecewise constant function. This type of front tracking methods has been developed in the context of gas dynamics by Holden, Lie, Risebro, Tveito, and coworkers [HLR99, RT92]. The latter class of front tracking methods without background grid is considered in the current chapter.

Front tracking methods in one dimension are based on the piecewise constant approximation of the solution. In the simulation of an initial-boundary 
value problem, the initial conditions are approximated by a piecewise constant function. At the discontinuities in this approximation, the flow conditions resemble the initial conditions of a local Riemann problem. After solving the local Riemann problems, their solutions are also approximated by a piecewise constant function. The position of the moving discontinuities in this approximation is tracked by the fronts. At an intersection of two fronts, the solution again resembles the initial condition of a local Riemann problem. The piecewise constant approximation of the solution of the local Riemann problem results in the creation of new fronts and so on until the solution in the space-time domain has been found.

One-dimensional front tracking is of interest for, for example, pipe flows and shock tube problems. A similar approach can also be employed to simulate two-dimensional supersonic flows. In addition, front tracking in one dimension can be used for a basic comparison of different front tracking algorithms and as a test for the implementation in a multidimensional code as a tool in dimensional splitting. Extensions of front tracking methods to multiple spatial dimensions can be found in for example [HLR99].

Due to the piecewise constant approximation of the solution, front tracking methods usually result in first-order error convergence [RT92]. An example of a second-order moving mesh method is the method by Lucier [Luc86] for scalar conservation laws.

In this chapter, a second-order front tracking method for the system of Euler equations is proposed. In [Wit06], it has been demonstrated numerically that the position of the fronts is approximated with second-order accuracy even for a first-order front tracking method. The first-order convergence is caused by the piecewise constant approximation of the solution. Therefore, a piecewise linear reconstruction of the solution of a first-order front tracking method can result in second-order error convergence. For a correct piecewise linear reconstruction, wave types are employed which track the physical phenomena that the fronts represent. In [WKB06], these wave types are used for an improved front interaction modeling, which results in a physically more correct simulation.

This chapter is organized as follows. In Sect. 2, the second-order improved front tracking method is introduced. In Sect. 3, numerical results are compared with those of the Godunov method and experimental data. The conclusions are summarized in Sect. 4.

\section{A Second-Order Front Tracking Method}

In this section, a second-order improved front tracking method for the Euler equations is proposed based on a piecewise linear reconstruction of the solution of a first-order front tracking method. For the reconstruction the wave types of the improved front tracking method [WKB06] are employed. It is 
demonstrated in numerical experiments in Sect. 3 that the piecewise linear solution results in second-order error convergence.

\subsection{Wave Types}

In standard front tracking methods without background grid, the front interactions are resolved by solving standard local Riemann problems at the intersection points. This approach has some limitations; for example, it cannot resolve isentropic compressions as truly isentropic phenomena. In [WKB06], an improved front interaction modeling is proposed, which employs wave front types to track the physical phenomena that the fronts represent for a physically more correct modeling of the Euler equations. The wave types are also employed for neglecting insignificant waves to avoid the build up of an infinite number of fronts. The same wave type labels are employed here for a piecewise linear reconstruction of the solution.

The basic waves in an Euler flow are shock waves, rarefaction or isentropic compression waves, and contact waves. For a detailed review of wave phenomena and classifications, the reader is referred to standard works as [CM79, Smo94].

In the front tracking method, the discontinuous phenomena such as a shock wave and a contact discontinuity are discretized by a single discontinuity. The wave front types that are assigned to the fronts representing these discontinuities are the shock wave (sw) and contact discontinuity (cd) wave types, respectively.

The continuous phenomena such as a rarefaction or isentropic compression wave are discretized by a series of small discontinuities. The fronts discretizing these continuous phenomena resemble characteristics. To represent both the solution inside the rarefaction fan and its spatial dimension accurately, three characteristic wave types are used: the left most characteristic (lch), the right most characteristic ( $\mathrm{rch}$ ), and an interior characteristic (ich) of a fan of characteristics. The same wave types are used in discretizing isentropic compression waves.

In contrast with a discontinuous change of entropy at a contact discontinuity, a continuous change of entropy is discretized by a series of contact waves. They are represented by similar wave types as rarefaction fans: the left most contact wave (lcw), the right most contact wave ( $\mathrm{rcw})$, and an interior contact wave (icw) of a continuous change in entropy.

Finally, a wave family type is assigned to the fronts to track the velocity of the wave relative to the flow. Left running $(-1)$, right running $(+1)$, and waves with no relative velocity with respect to the flow (0) are used.

\subsection{Piecewise Linear Reconstruction}

A piecewise linear reconstruction of the solution of a front tracking method is not straightforward due to the large variations in cell size from a cell to 
another. Based on the wave types, it can be identified whether the flow conditions in a cell are truly uniform or not. In the latter case, a piecewise linear reconstruction of the flow conditions is used based on the wave types.

Consider the piecewise constant solution of the improved front tracking method with $n_{\mathrm{f}}$ fronts and $n_{\Omega}$ cells in space-time. Let $f_{j}$ denote the fronts and $\Omega_{i}$ denote the cells with flow conditions $U_{i}$ in the space-time solution. Then the cell numbers of the cell to the left and the right of front $f_{j}$ are $i_{\mathrm{f}_{j}, \mathrm{l}}$ and $i_{\mathrm{f}_{j}, \mathrm{r}}$, respectively. The numbers of the fronts to the left and the right of cell $\Omega_{i}$ are $j_{\Omega_{i}, 1}(t)$ and $j_{\Omega_{i}, \mathrm{r}}(t)$, respectively.

Consider the simple example of a single discontinuity in the initial condition at which only a centered rarefaction fan is created (see Fig.1). The piecewise constant approximation results in $n_{\Omega}$ uniform domains separated by $n_{\mathrm{f}}=n_{\Omega}-1$ discontinuities. At the local Riemann problem, the created cells are labeled as domains with truly uniform or nonuniform flow conditions based on the position of the cell in the solution of the Riemann problem. In this case, the flow conditions in cells $\Omega_{1}$ and $\Omega_{n_{\Omega}}$ are labeled as uniform, where the cells are numbered from the left to the right. These cells are not affected by the piecewise linear reconstruction. In the other cells $\Omega_{i}$ with $i=2, \ldots, n_{\Omega}-1$, which discretize the rarefaction wave, the flow conditions are labeled as nonuniform.

Front $f_{1}$ in Fig. 1 has wave type lch, front $f_{n_{\mathrm{f}}}$ has wave type rch, and fronts $f_{j}$ with $j=2, \ldots, n_{\mathrm{f}}-1$ have wave type ich, where the fronts are numbered from the left to the right. These wave types are used in the piecewise linear reconstruction of the solution in the cells $\Omega_{i}$ with $i=2, \ldots, n_{\Omega}-1$. At all characteristic wave types, the flow conditions vary continuously. At front $f_{1}$ with wave type lch, the flow conditions are equal to those in the cell to its left $U_{i_{\mathrm{f}_{1}, 1}}$. The flow conditions at front $f_{n_{\mathrm{f}}}$ with wave type rch are equal to those in the cell to its right $U_{i_{\mathrm{f}_{\mathrm{f}},} \text {. }}$. At the fronts with wave type ich, $f_{j}$ with $j=2, \ldots, n_{\mathrm{f}}-1$, the flow conditions are the average of those in the cell to its left and its right $\left(U_{i_{\mathrm{f}_{j}, 1}}+U_{i_{\mathrm{f}_{j}, \mathrm{r}}}\right) / 2$.

Since the gradient of the flow conditions changes in time and in general, the cells $\Omega_{i}$ can be bounded by different fronts $f_{j_{\Omega_{i}, 1}(t)}$ and $f_{j_{\Omega_{i}, \mathrm{r}}(t)}$ in time, the piecewise linear flow conditions in the cells $\Omega_{i}$ with $i=2, \ldots, n_{\Omega}-1$ are represented as follows. The flow velocity in a centered rarefaction wave, which originates in a single point, varies exactly linear in space. Therefore, linear flow conditions in the cells $\Omega_{i}$ are reconstructed as if the cell is a centered
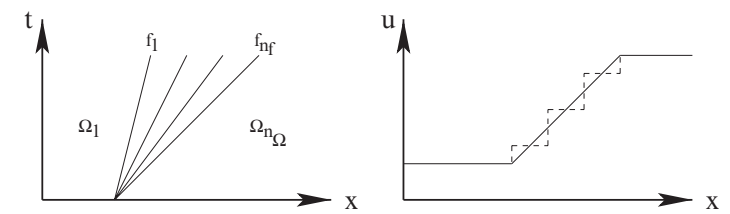

Fig. 1. The piecewise linear reconstruction of the solution of a rarefaction fan 
rarefaction wave with origin $\mathcal{O}_{i}$ at $\left(x_{\mathcal{O}_{i}}, t_{\mathcal{O}_{i}}\right)$. The origin of the centered rarefaction wave might be the actual origin of the cell or a virtual origin. The flow conditions at the fronts bounding the cell initially at the left and the right are linearly projected onto flow conditions at lines with velocity $u^{-}=-1$ and $u^{+}=1$ through the cell origin $\mathcal{O}_{i}, U_{i}^{-}$and $U_{i}^{+}$, respectively. The piecewise linear flow conditions $U_{i}(x, t)$ in cell $\Omega_{i}$ are then based on the linear relation through $U_{i}^{-}$and $U_{i}^{+}$:

$$
U_{i}(x, t)=\frac{1}{2}\left(U_{i}^{+}+U_{i}^{-}\right)+\frac{1}{2}\left(U_{i}^{+}-U_{i}^{-}\right) \frac{x-x_{\mathcal{O}_{i}}}{t-t_{\mathcal{O}_{i}}},
$$

with $(x, t) \in \Omega_{i}$. The result of this linearization is for the centered rarefaction fan shown in Fig. 1. This piecewise linear reconstruction can mostly be employed as a postprocessing step. Special attention has to be paid at the cells at the final time level and the piecewise linear reconstruction of the flow conditions in nonsimple waves.

\section{Numerical Results}

In this section, the second-order front tracking method is verified by comparison of numerical results with those of Godunov method for a one-dimensional unsteady Riemann problem. A validation study is performed by comparing results for a supersonic wing section flow with experimental data.

\subsection{Verification}

The second-order front tracking method is applied to Sod's Riemann problem [Sod78] to verify the convergence in comparison with the first-order Godunov finite volume method [Tor97]. The initial left and right states of Sod's Riemann problem are defined as $p_{\text {left }}=\rho_{\text {left }}=1, p_{\text {right }}=0.1$, $\rho_{\text {right }}=0.125$, and zero velocity.

In Fig. 2, the solution for the density at $t=1$ is given for the front tracking (FT) method with $n_{\mathrm{f}}=8$ and for the finite volume (FV) method with $n_{\mathrm{x}}=128$ spatial cells, and $\mathrm{cfl}=0.5$. The front tracking method results in an approximation of the shock wave and the contact discontinuity as true discontinuities, since the uniform domains in the solution are unaffected by the piecewise linear reconstruction. The flow conditions in the rarefaction fan are approximated accurately by the piecewise linear representation using eight characteristics. The most profound difference with the finite volume results is the numerical smearing of especially the contact discontinuity in the finite volume results.

The $L_{1}$ error convergence with respect to $n_{\mathrm{f}}$ and $n_{\mathrm{x}}$ is shown in Fig. 3 for the piecewise constant and piecewise linear front tracking method, and the finite volume method. The piecewise linear reconstruction of the solution 


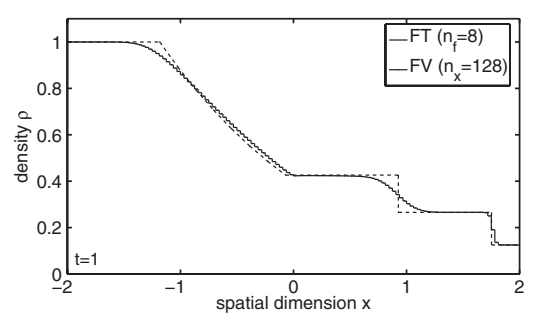

Fig. 2. Front tracking (FT) and finite volume (FV) solution for the density of Sod's Riemann problem at $t=1$

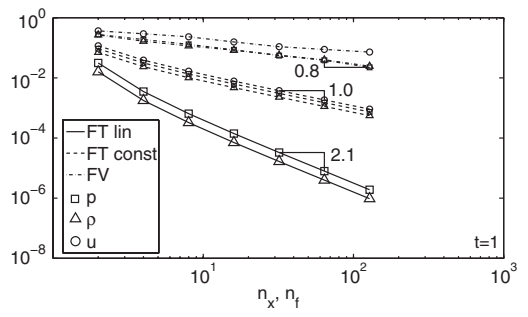

Fig. 3. Error convergence of the front tracking (FT) method and the finite volume (FV) method for Sod's Riemann problem at $t=1$

results in second-order error convergence for the front tracking method. This results in significantly lower error than for the first-order piecewise constant front tracking method. The finite volume method converges approximately with first-order accuracy toward the front tracking solution. The absolute error is, however, larger than for the first-order front tracking method due to the numerical smearing of the contact discontinuity.

\subsection{Validation}

A similar front tracking algorithm as for one-dimensional unsteady Euler flow can also be applied to two-dimensional supersonic flow problems. Supersonic flow over a circular arc wing section with a maximum thickness of $12 \%$ in a free stream flow with Mach number 2 and 2.5 is considered. The numerical results for the drag of the wing section are validated by comparison with experimental data.

In Figs. 4 and 5, the front tracking solution for the Mach number field is shown for free stream Mach numbers of $M_{\infty}=2$ and $M_{\infty}=2.5$, respectively. The surface of the wing section is approximated by $n_{\mathrm{f}}=41$ points. The leading and trailing edge shock wave are slightly curved due to their interaction with the expansion created at the wing section surface. The front tracking method also resolves a slight gradient of the Mach number downstream of the trailing edge shock wave due to the entropy gradient caused by the curved shocks. 

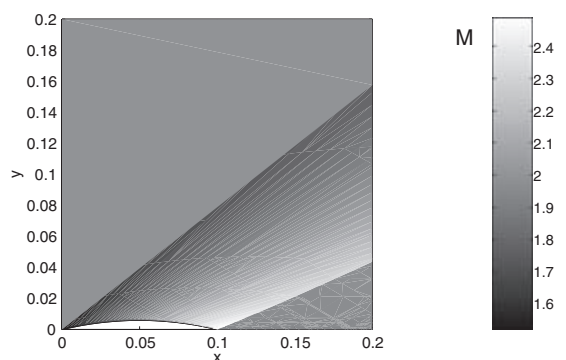

Fig. 4. Front tracking solution for the Mach number field of the supersonic wing section flow with $M_{\infty}=2$
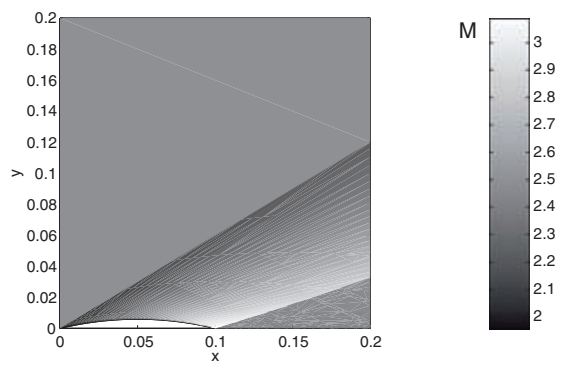

Fig. 5. Front tracking solution for the Mach number field of the supersonic wing section flow with $M_{\infty}=2.5$

Table 1. Drag of the supersonic wing section obtained from front tracking simulations and experiments

\begin{tabular}{|c|c|c|}
\hline Mach & Cells Compu & Measured \\
\hline 2 & $2,0280.0450$ & 0.0462 \\
\hline 2.5 & $8,142 \quad 0.0339$ & 0.0330 \\
\hline
\end{tabular}

The case of $M_{\infty}=2.5$ in Fig. 5 results in a slightly higher range of Mach numbers and steeper shock wave angles compared to $M_{\infty}=2$ in Fig. 4 .

In Table 1, the drag computed by the front tracking method is compared with experimental data. The experimental data are obtained by pressure hole measurements performed by Souverein, Van Oudheusden, and Scarano [Sou77, SOS07]. The numerical results for the drag show good agreement with the measured values for both $M_{\infty}=2$ and $M_{\infty}=2.5$.

\section{Conclusions}

A piecewise linear reconstruction of the solution of the improved front tracking method is proposed. A comparison with the Godunov method demonstrated 
second-order error convergence for Sod's Riemann problem. The results of the application to two-dimensional supersonic wing section flow showed good agreement with experimental data. More challenging test problems including multidimensional unsteady problems will be considered in future work.

\section{References}

[CGM86] Chern, I.-L., Glimm, J., McBryan, O., Plohr, B., Yaniv, S., Front tracking for gas dynamics, J. Comput. Phys., 62, 83 (1986)

[CM79] Chorin, A.J., Marsden, J.E.: A mathematical introduction to fluid mechanics. Springer-Verlag, New York (1979)

[HLR99] Holden, H., Lie, K.-A., Risebro, N.H.: An unconditionally stable method for the Euler equations, J. Comput. Phys., 150, 76 (1999)

[HGS95] Holmes, R.L., Grove, J.W., Sharp, D.H.: Numerical investigation of Richtmyer-Meshkov instability using front tracking, J. Fluid Mech., 301, $51(1995)$

[Luc86] Lucier, B.J.: A moving mesh numerical method for hyperbolic conservation laws, Math. Comp., 173, 59 (1986)

[Mor87] Moretti, G.: Computations of flows with shocks. Ann. Rev. Fluid Mech., 19, 313 (1987)

[RM67] Richtmyer, R., K. Morton: Difference methods for intial value problems. Interscience, New York (1967)

[RT92] Risebro, N.H., Tveito, A.: A front tracking method for conservation laws in one dimension, J. Comput. Phys., 101, 130 (1992)

[Smo94] Smoller, J.: Shock waves and reaction-diffusion equations (2nd ed) Springer-Verlag, New York (1994)

[Sod78] Sod, G.A.: A survey of several finite difference methods for systems of nonlinear hyperbolic conservation laws, J. Comput. Phys., 27, 1 (1978)

[Sou77] Souverein, L.J.: PIV based aerodynamic loads determination in supersonic flows. MSc Thesis, Delft University of Technology, Delft (2006)

[SOS07] Souverein, L.J., van Oudheusden, B.W., Scarano, F.: Particle image velocimetry based loads determination in supersonic flows. AIAA-2007-50, 45th AIAA Aerospace Sciences Meeting and Exhibit, Reno, NV (2007)

[SW86] Swartz, B.K., Wendroff, B.,: AZTEC: A front tracking code based on Godunov's method. Appl. Numer. Math., 2, 385 (1986)

[Tor97] Toro, E.F.: Riemann solvers and numerical methods for fluid dynamics, Springer Verlag, Berlin (1997)

[WKB06] Witteveen, J.A.S., Koren, B., Bakker, P.G.: An improved front tracking method for the Euler equations. J. Comput. Phys., in press

[Wit06] Witteveen, J.A.S.: A second-order front tracking method applied to the Euler equations. AIAA-2006-1277, 44th AIAA Aerospace Sciences Meeting and Exhibit, Reno, NV (2006) 